\title{
Src kinase inhibition with dasatinib impairs neutrophil function and clearance of Escherichia coli infection in a murine model of acute lung injury
}

James G. Macfarlane ${ }^{1 *}$ (D), David A. Dorward², Marie-Hélène Ruchaud-Sparagano ${ }^{1}$, Jonathan Scott', Christopher D. Lucas², Adriano G. Rossi ${ }^{2}$ and A. John Simpson ${ }^{1}$

\begin{abstract}
Background: Neutrophils rapidly respond to and clear infection from tissues, but can also induce tissue damage through excessive degranulation, when acute inflammation proceeds unchecked. A number of key neutrophil functions, including adhesion-dependent degranulation, are controlled by src family kinases. Dasatinib is a potent src inhibitor used in treating patients with chronic myeloid leukaemia and treatment-resistant acute lymphoblastic leukaemia. We hypothesized that dasatinib would attenuate acute inflammation by inhibiting neutrophil recruitment, degranulation and endothelial cell injury, without impairing bacterial clearance, in a murine model of bacteria-induced acute lung injury. C57BL/6 mice received intratracheal Escherichia coli, and were treated with intraperitoneal dasatinib or control. Bacterial clearance, lung injury, and markers of neutrophil recruitment and degranulation were measured. Separately, human blood neutrophils were exposed to dasatinib or control, and the effects on a range of neutrophil functions assessed.
\end{abstract}

Results: Dasatinib was associated with a dose-dependent significant increase in E. coli in the mouse lung, accompanied by impairment of organ function, reflected in significantly increased protein leak across the alveolarcapillary membrane. However, the number of neutrophils entering the lung was unaffected, suggesting that dasatinib impairs neutrophil function independent of migration. Dasatinib did not cause direct toxicity to human neutrophils, but led to significant reductions in phagocytosis of $E$. coli, adhesion, chemotaxis, generation of superoxide anion and degranulation of primary and secondary granules. However, no biologically important effect of dasatinib on neutrophil degranulation was observed in mice.

Conclusions: Contrary to our starting hypothesis, src kinase inhibition with dasatinib had a detrimental effect on bacterial clearance in the mouse lung and therefore does not represent an attractive therapeutic strategy to treat primary infective lung inflammation. Data from human neutrophils suggest that dasatanib has inhibitory effects on a range of neutrophil functions.

Keywords: Dasatinib, Src kinases, Neutrophil, Acute lung injury, acute respiratory distress syndrome, Pneumonia, Phagocytosis, Infection

\footnotetext{
* Correspondence: jim.macfarlane@nhs.net

'Translational and Clinical Research Institute, Newcastle University, Newcastle upon Tyne NE2 4HH, UK

Full list of author information is available at the end of the article
}

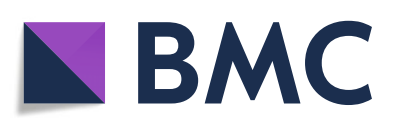

(- The Author(s). 2020 Open Access This article is licensed under a Creative Commons Attribution 4.0 International License, which permits use, sharing, adaptation, distribution and reproduction in any medium or format, as long as you give appropriate credit to the original author(s) and the source, provide a link to the Creative Commons licence, and indicate if changes were made. The images or other third party material in this article are included in the article's Creative Commons licence, unless indicated otherwise in a credit line to the material. If material is not included in the article's Creative Commons licence and your intended use is not permitted by statutory regulation or exceeds the permitted use, you will need to obtain permission directly from the copyright holder. To view a copy of this licence, visit http://creativecommons.org/licenses/by/4.0/. The Creative Commons Public Domain Dedication waiver (http://creativecommons.org/publicdomain/zero/1.0/) applies to the data made available in this article, unless otherwise stated in a credit line to the data. 


\section{Background}

Neutrophils are key cellular mediators involved in rapidly responding to and clearing bacterial and fungal infection. They are recruited early to the alveoli of patients with pulmonary sepsis, acute lung injury (ALI) and acute respiratory distress syndrome (ARDS) and their levels correlate with degree of hypoxia, protein leak and clinical outcome [1-3]. Early activation is achieved by coordinated retention of circulating neutrophils by endothelial cells, followed by neutrophil extravasation, chemotaxis towards pathogens, and ingestion of these pathogens in discrete phagosomes [4, 5]. Following phagocytosis, neutrophil granules containing proteolytic enzymes and machinery to generate reactive oxygen species fuse with the phagosome, and killing of pathogens proceeds [6-8]. Local, extracellular release of granule products is potently anti-microbial and proinflammatory, and can therefore be considered as a physiological response when regulated. However, depending on the size of the inflammatory stimulus and the intensity of the neutrophil response, excessive neutrophil granule release may become pathological, with neutrophil proteases, cytokines and reactive oxygen species (ROS) capable of direct and indirect damage to the alveolar epithelium, leading to increased permeability and protein-rich fluid leak into the alveolar space $[9,10]$.

Several key neutrophil functions are regulated by src kinases. Adhesion-dependent neutrophil degranulation is regulated by the src kinases Hck and Fgr [11]. Neutrophils from mice deficient in Hck, Fgr and Lyn fail to degranulate or generate superoxide anion in response to the bacterial virulence factor formyl methionine leucine phenylalanine (fMLF) [12]. Activation of neutrophils via cell surface Fc gamma receptors is also impaired in vivo in Hck/Fgr/Lyn-null mice [13].

Dasatinib is a src kinase inhibitor that is widely used in the treatment of Philadelphia chromosome-positive chronic myeloid leukemia (CML) and treatmentresistant acute lymphoblastic leukaemia (ALL), with the potential for use in other malignancies being actively explored $[14,15]$. In vitro, dasatinib blocks adhesiondependent neutrophil degranulation, with little effect on chemotaxis, phagocytosis or bacterial killing at serum concentrations of $50-100 \mathrm{nM}$, similar to physiological concentrations measured in dasatinib-treated CML patients [16]. In a murine lipopolysaccharide (LPS)-induced model of acute lung injury, dasatinib attenuated lung inflammation but its effect was dependent on the dose and the method of LPS delivery [17]. In the caecal ligation and puncture (CLP) model of sepsis, pre- and post-CLP administration of low dose dasatinib $(1 \mathrm{mg} / \mathrm{kg})$ improved survival by $50 \%$ whereas survival was worsened by a higher dose $(10 \mathrm{mg} / \mathrm{kg})$ [18].
Based on these in vitro and in vivo data, our starting hypothesis was that src kinase inhibition with dasatinib would prove beneficial in infective ALI/ARDS, by attenuating neutrophilic inflammation, while maintaining sufficient neutrophil phagocytic and killing capacity to clear infection. Until now the effect of dasatinib in models of direct bacterial lung infection has been poorly characterized. We therefore sought to test our hypothesis in an in vivo model of bacteria-induced acute lung injury. As described below, our data did not support our starting hypothesis, instead suggesting that dasatinib is associated with reduced bacterial clearance.

\section{Results}

Dasatanib impairs lung clearance of $E$. coli infection and increases alveolar leak

Experiments were performed to establish the time course of E. coli-mediated recruitment of neutrophils to the alveolar space and pulmonary interstitium (see Supplementary Fig. 1, Additional file 1). A control experiment with dasatinib alone at a dose of $1 \mathrm{mg} / \mathrm{kg}$ or $10 \mathrm{mg} / \mathrm{kg}$, without E. coli, showed that dasatinib itself did not cause alveolar protein leak or increased cellular influx to the alveolar space (Supplementary Fig. 2, Additional File 1). All subsequent in vivo experiments examined outcome measures $24 \mathrm{~h}$ after intratracheal (i.t.) instillation of $1 \times 10^{6}$ colony forming units (cfu) E. coli.

Intraperitoneal (i.p.) dasatinib treatment at $0 \mathrm{~h}$ and $12 \mathrm{~h}$ was associated with a dose-dependent increase in the concentration of bacteria retrieved from the lungs of mice after i.t. administration of $E$. coli (delivered $30 \mathrm{~min}$ after the first dasatanib treatment), as compared to i.p. vehicle control (Fig. 1). Extra-pulmonary isolation of E. coli was observed in a small number of mice treated with the higher dose of dasatinib, $10 \mathrm{mg} / \mathrm{kg}$. (Fig. 1).

The increased pulmonary bacterial load observed after treatment with dasatinib at a dose of $10 \mathrm{mg} / \mathrm{kg}$ was associated with reduced barrier function of the alveolarcapillary membrane, as evidenced by significantly increased concentrations of total protein in bronchoalveolar lavage (BAL) fluid obtained $24 \mathrm{~h}$ after instillation of bacteria (Fig. 2a). This observation was verified by measuring IgM, as an example of a large molecule, the concentration of which is closely regulated in BAL fluid (Fig. 2b).

\section{Dasatinib has no effect on neutrophil recruitment or degranulation}

Disruption to the alveolar-capillary membrane can be caused by infiltration of activated leukocytes [9, 10, 19]. However, dasatinib had no influence on the proportion of leukocytes in BAL fluid, which was consistently 85\% leucocytes and $\sim 15 \%$ alveolar macrophages in control, 1 $\mathrm{mg} / \mathrm{kg}$ or $10 \mathrm{mg} / \mathrm{kg}$ dasatinib-treated mice, after E. coli 

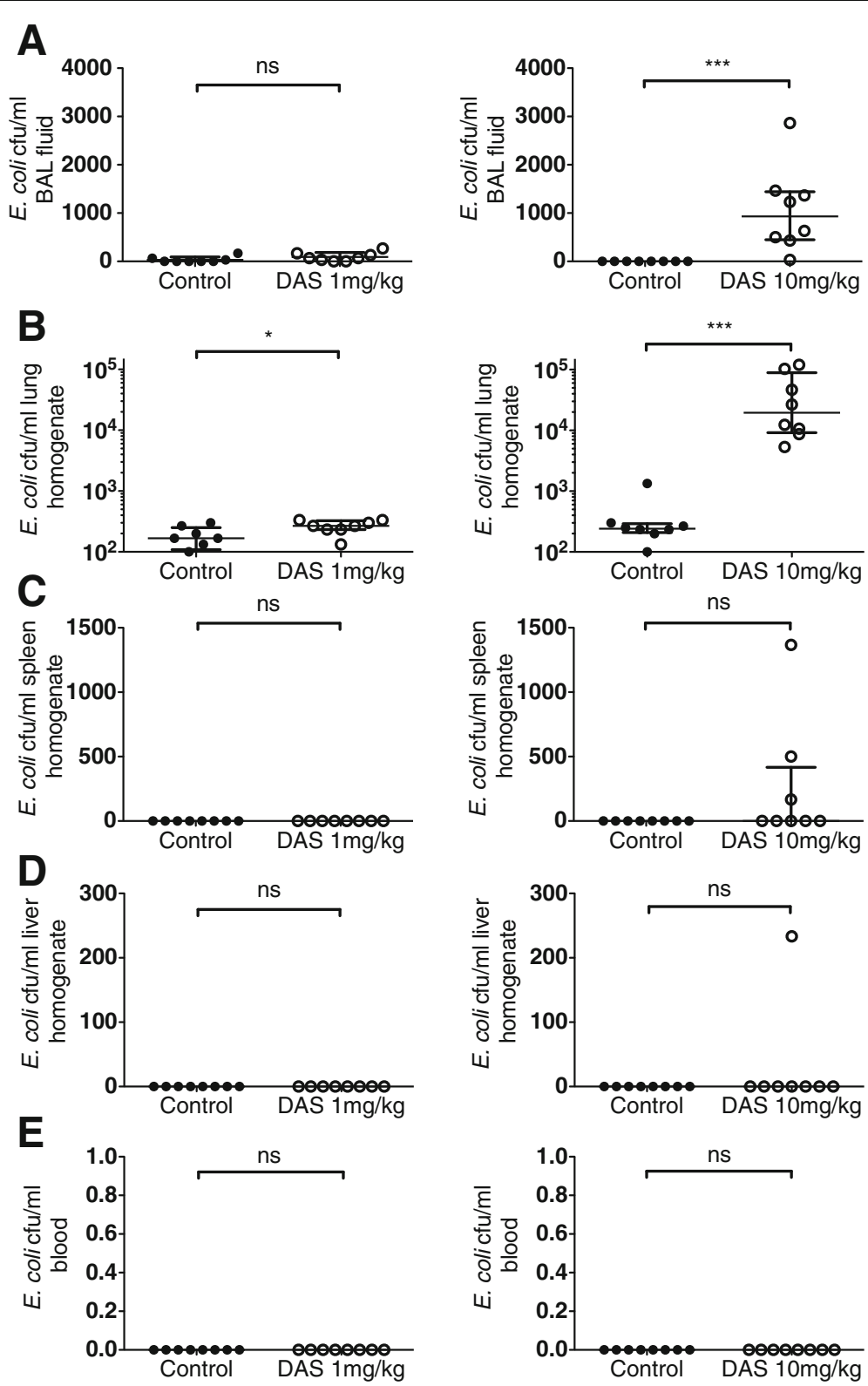

Fig. 1 Effect of dasatinib pre-treatment on E. coli presence and survival in $\mathbf{a}$, BAL fluid, $\mathbf{b}$, lung homogenates, $\mathbf{c}$, spleen, $\mathbf{d}$, liver and $\mathbf{e}$, peripheral blood from mice exposed to i.t. E. coli. ${ }^{*} p<0.05,{ }^{* * *} p<0.001$. Values were derived from two experiments ( $1 \mathrm{mg} / \mathrm{kg}$ or $10 \mathrm{mg} / \mathrm{kg}$ dasatinib vs. control administered at 0 and $12 \mathrm{~h}$ ) using 8 mice per group. DAS = dasatinib

treatment (Fig. 3). In keeping with this, concentrations of pro- and anti-inflammatory cytokines in BAL fluid were similar in the dasatinib and control groups, with small increments in mean levels of keratinocyte chemoattractant $(\mathrm{KC})$ and tumour necrosis factor (TNF) in BAL fluid from mice treated with dasatinib at $10 \mathrm{mg} / \mathrm{kg}$ (see Supplementary Fig. 3 , Additional file 1). No differences were observed in the number of pulmonary interstitial neutrophils in the dasatinib and control groups (see Supplementary Fig. 4, Additional file 1).
Dasatinib appeared to have a minimal effect on degranulation of neutrophils in vivo (Fig. 4). No difference in expression of neutrophil CD11b was found (Fig. 4a). Myeloperoxidase (MPO) activity was increased after treatment with dasatinib at a dose of $10 \mathrm{mg} / \mathrm{kg}$, with no effect on concentrations of extracellular MPO antigen (Fig. 4b and c).

\section{Dasatinib increases extrapulmonary toxicity}

Extrapulmonary toxicity also appeared to be increased by the higher concentration of dasatinib $(10 \mathrm{mg} / \mathrm{kg})$, 

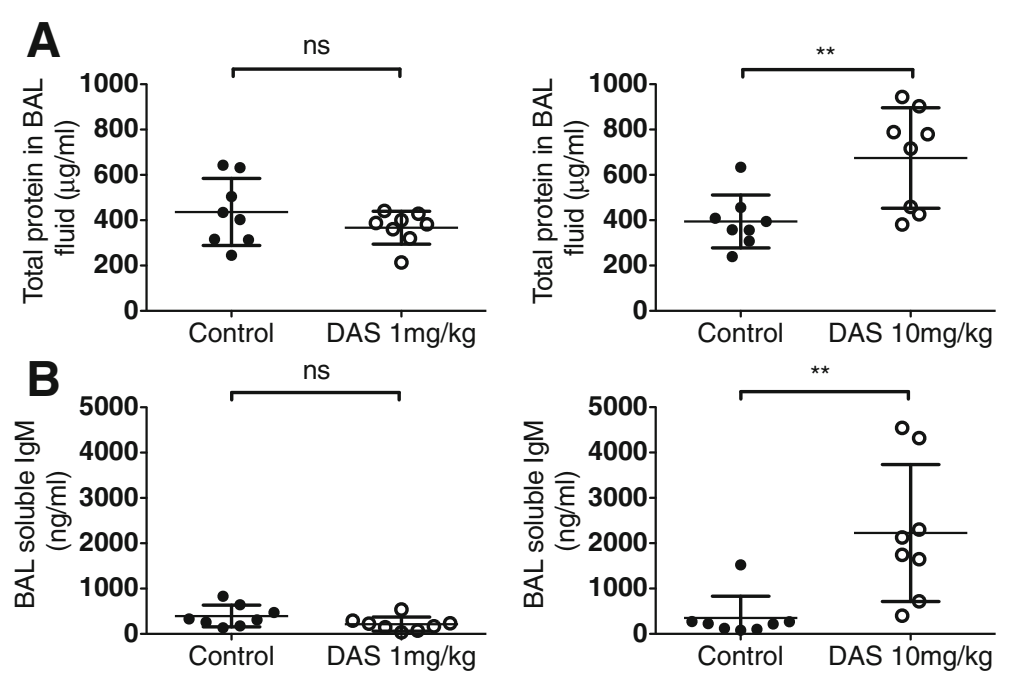

Fig. 2 Effect of dasatinib pre-treatment on alveolar protein leak. a Total protein in BAL fluid. b Soluble IgM in BAL fluid from mice exposed to i.t. E. coli. ${ }^{* *} p<0.01$. Values were derived from two experiments $(1 \mathrm{mg} / \mathrm{kg}$ or $10 \mathrm{mg} / \mathrm{kg}$ dasatinib vs. control administered at 0 and $12 \mathrm{~h}$ ) using 8 mice per group
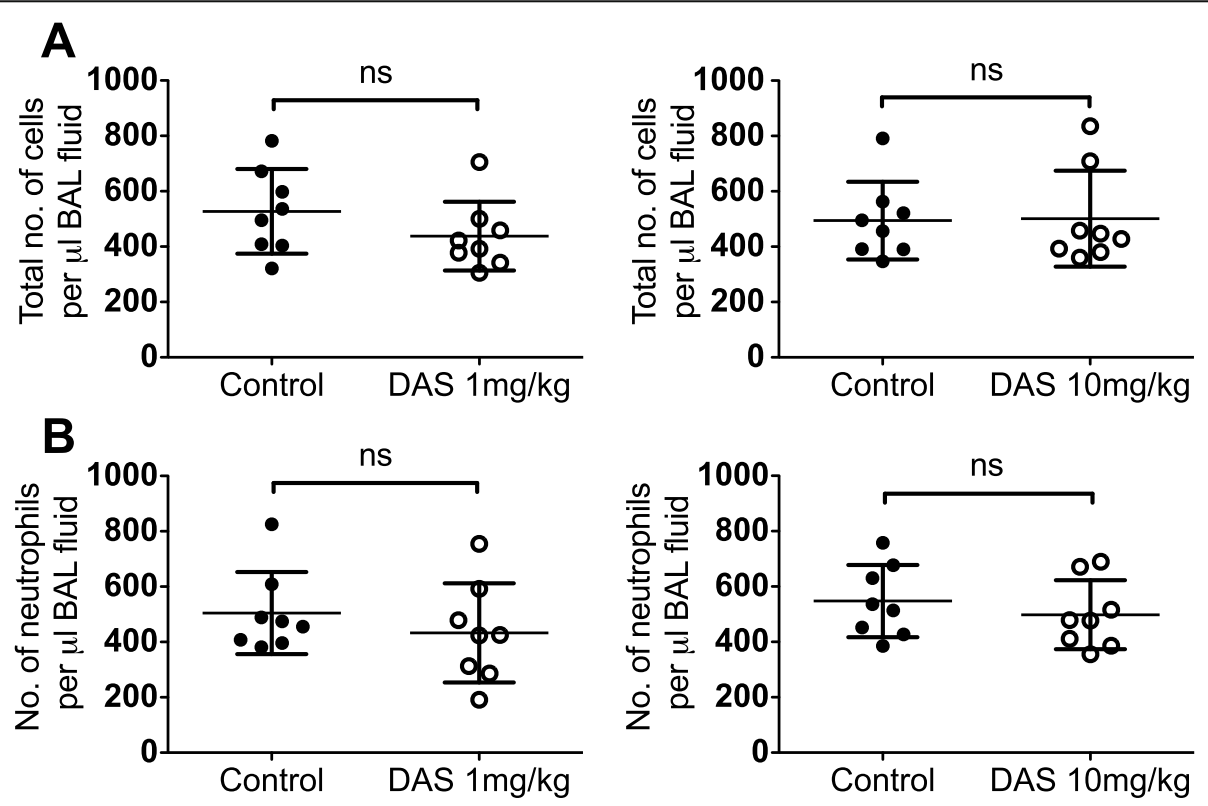

\section{C}
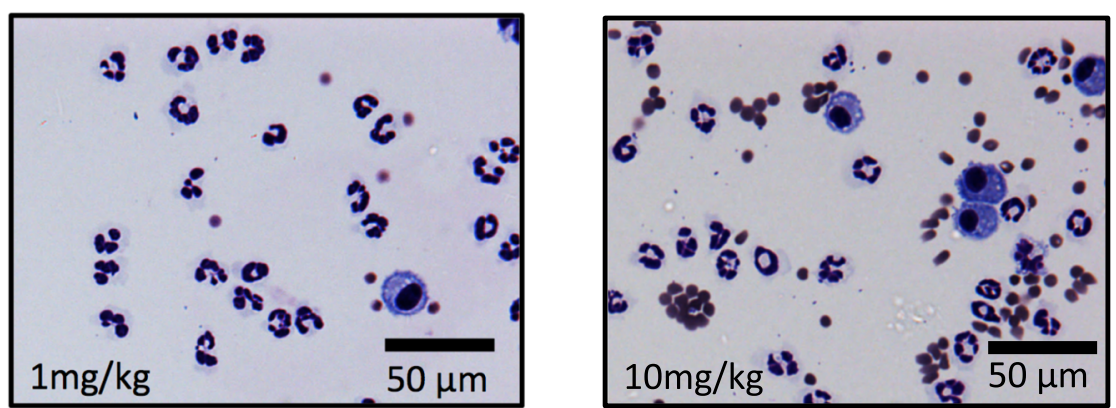

Fig. 3 Effect of dasatinib pre-treatment on cellular influx into the alveolar space. a Total number of cells in BAL fluid. b Total number of neutrophils in BAL fluid. c Representative cytocentrifuge preparations of BAL fluid (400x magnification) from mice exposed to i.t. E. coli. Values were derived from two experiments ( $1 \mathrm{mg} / \mathrm{kg}$ or $10 \mathrm{mg} / \mathrm{kg}$ dasatinib vs. control administered at 0 and $12 \mathrm{~h}$ ) using 8 mice per group 

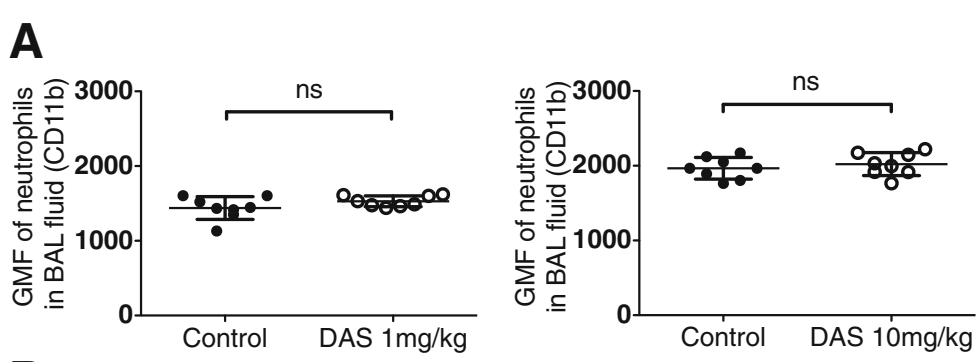

B
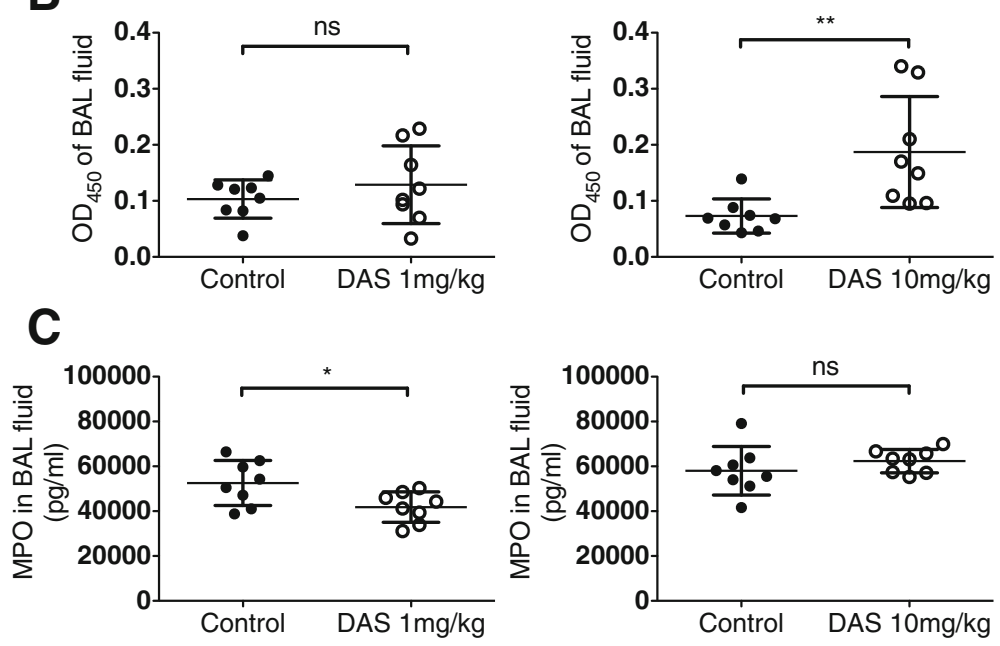

Fig. 4 Effect of dasatinib pre-treatment on markers of neutrophil degranulation in the alveolar space. a CD11b expression on BAL neutrophils, $\mathbf{b}$ MPO activity and $\mathbf{c}$ MPO concentration in BAL fluid from mice exposed to i.t. E. coli. ${ }^{*} p<0.05$, ${ }^{* *} p<0.01$. Values were derived from two experiments ( $1 \mathrm{mg} / \mathrm{kg}$ or $10 \mathrm{mg} / \mathrm{kg}$ dasatinib vs. control administered at 0 and $12 \mathrm{~h}$ ) using 8 mice per group. GMF = geometric mean fluorescence

which was associated with a consistent increase in plasma levels of the hepatic enzyme alanine transaminase (ALT), and a small but statistically significant increase in concentrations of plasma lactate (see Supplementary Fig. 5, Additional file 1).
Dasatinib inhibits a number of key neutrophil functions in vitro

In an attempt to determine whether subtle effects on neutrophil function may contribute to the failure of bacterial clearance in vivo, human neutrophils isolated from
A

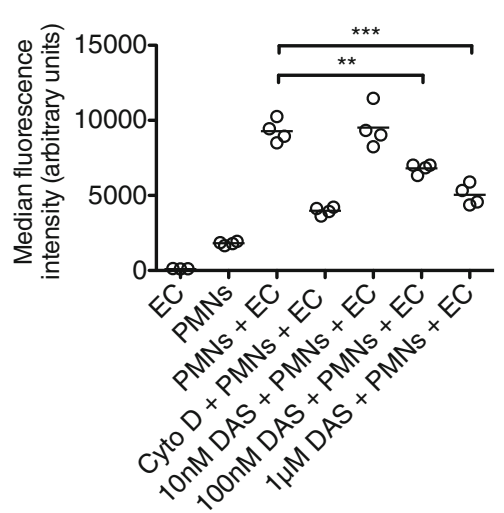

B

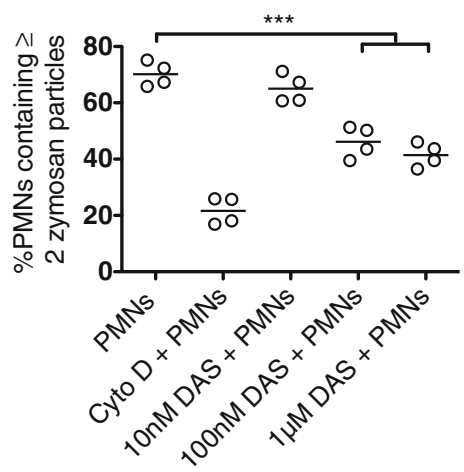

Fig. 5 In vitro phagocytosis by isolated human blood neutrophils pre-treated with dasatinib. a MFI values of adherent neutrophils exposed to pHrodo E. coli particles (EC) following pre-treatment with cytochalasin D or dasatinib. b Dose response of dasatinib treatment on neutrophil phagocytosis of autologous serum-opsonized zymosan particles. ${ }^{* *} p<0.001$. Values were derived from four experiments, each using neutrophils from different healthy volunteers 

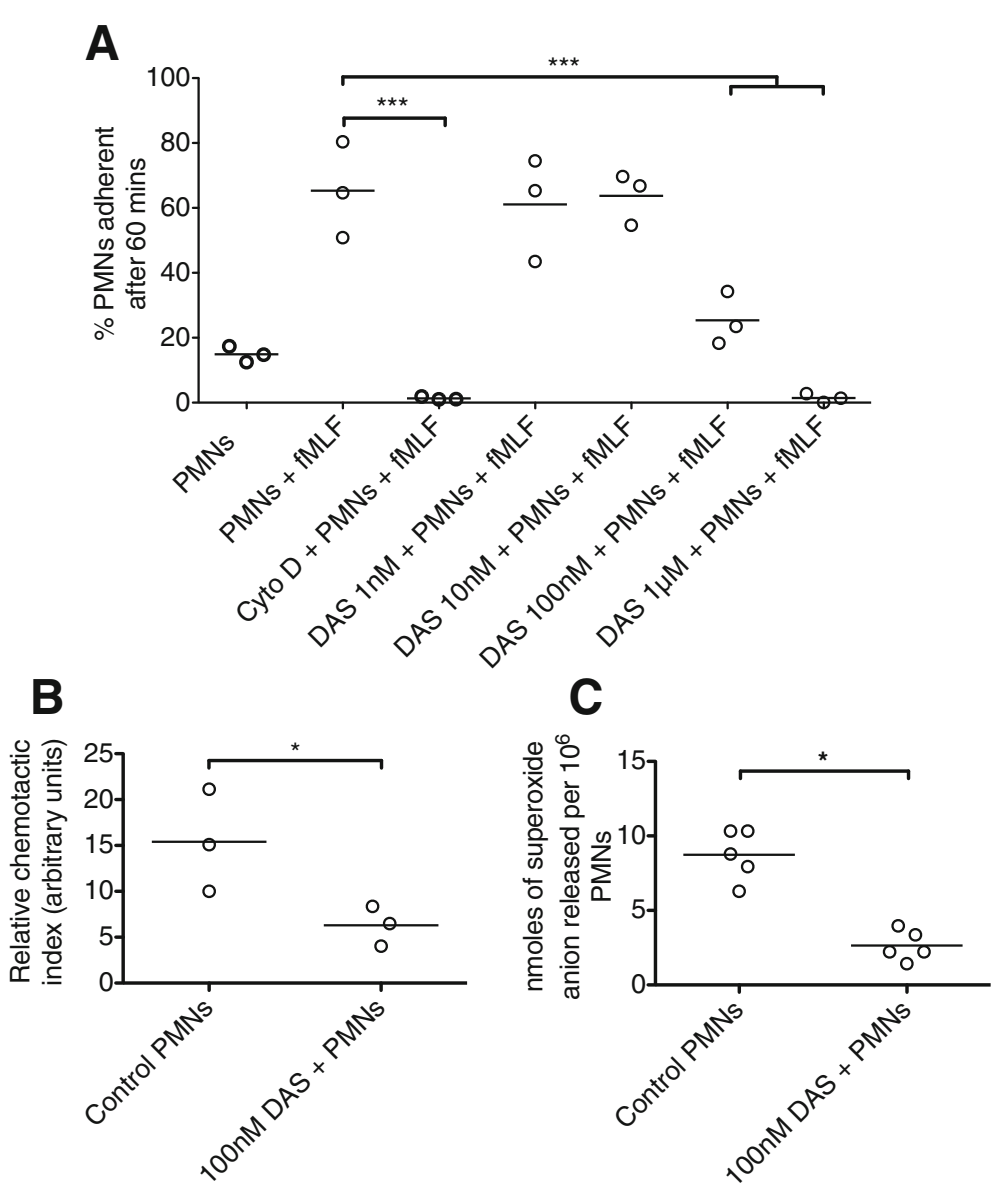

Fig. 6 Effect of dasatinib on isolated human blood neutrophil adherence, chemotaxis and superoxide production in vitro. a Relative adherence of stimulated neutrophils to tissue culture plastic and $\mathbf{b}$ relative 2-D chemotactic index of neutrophils towards formylated peptide, following pretreatment with dasatinib. $\mathbf{c}$ Extracellular superoxide production by stimulated neutrophils pre-treated with dasatinib. ${ }^{*} p<0.05,{ }^{* * *} p<0.001$. Values were derived from three $(A+B)$ or five $(C)$ experiments, each using neutrophils from different healthy volunteers

peripheral blood were exposed to dasatinib in vitro. Experiments were carried out to determine whether dasatinib had any direct toxic effects on neutrophils. Dasatinib had no influence on release of lactate dehydrogenase (LDH), or on neutrophil apoptosis (see Supplementary Fig. 6, Additional file 1).

Dasatinib treatment resulted in significant impairment in the capacity of neutrophils to phagocytose E. coli particles or zymosan (Fig. 5). Dasatinib was also associated with significant reductions in neutrophil adhesion, chemotaxis and superoxide anion generation (Fig. 6). Dasatinib had small absolute effects on neutrophil degranulation (including that induced by E. coli LPS), though at higher concentrations of dasatinib, statistically significant inhibition of CD63 (primary granule) and CD66b (secondary granule) was observed (Fig. 7).

\section{Discussion}

Our data indicate that src kinase inhibition with dasatinib is associated with dose-dependent impaired clearance of $E$. coli in vivo and in vitro. To our knowledge this is the first in vivo experimental demonstration modeling an increased susceptibility to pulmonary infection (with associated impairment of organ function) induced by dasatinib.

Our findings suggest that the failure to clear E. coli from the mouse lung is not attributable to impaired neutrophil recruitment, as the numbers of neutrophils entering the mouse lung were not significantly impaired by dasatinib. The in vitro experiments however suggested that neutrophil chemotaxis toward fMLF and adhesion to tissue culture plastic is inhibited by dasatinib. Several explanations could underlie the apparent discrepancy between the in vivo and in vitro findings for example, the range of chemotactic signals in vivo is likely to be far broader and more complex. Furthermore, the stimulus strength of live bacteria may be too potent for the doses of dasatinib reaching the lung in situ to inhibit chemotaxis, adhesion and migration significantly. Ultimately we feel that an effect of dasatinib 


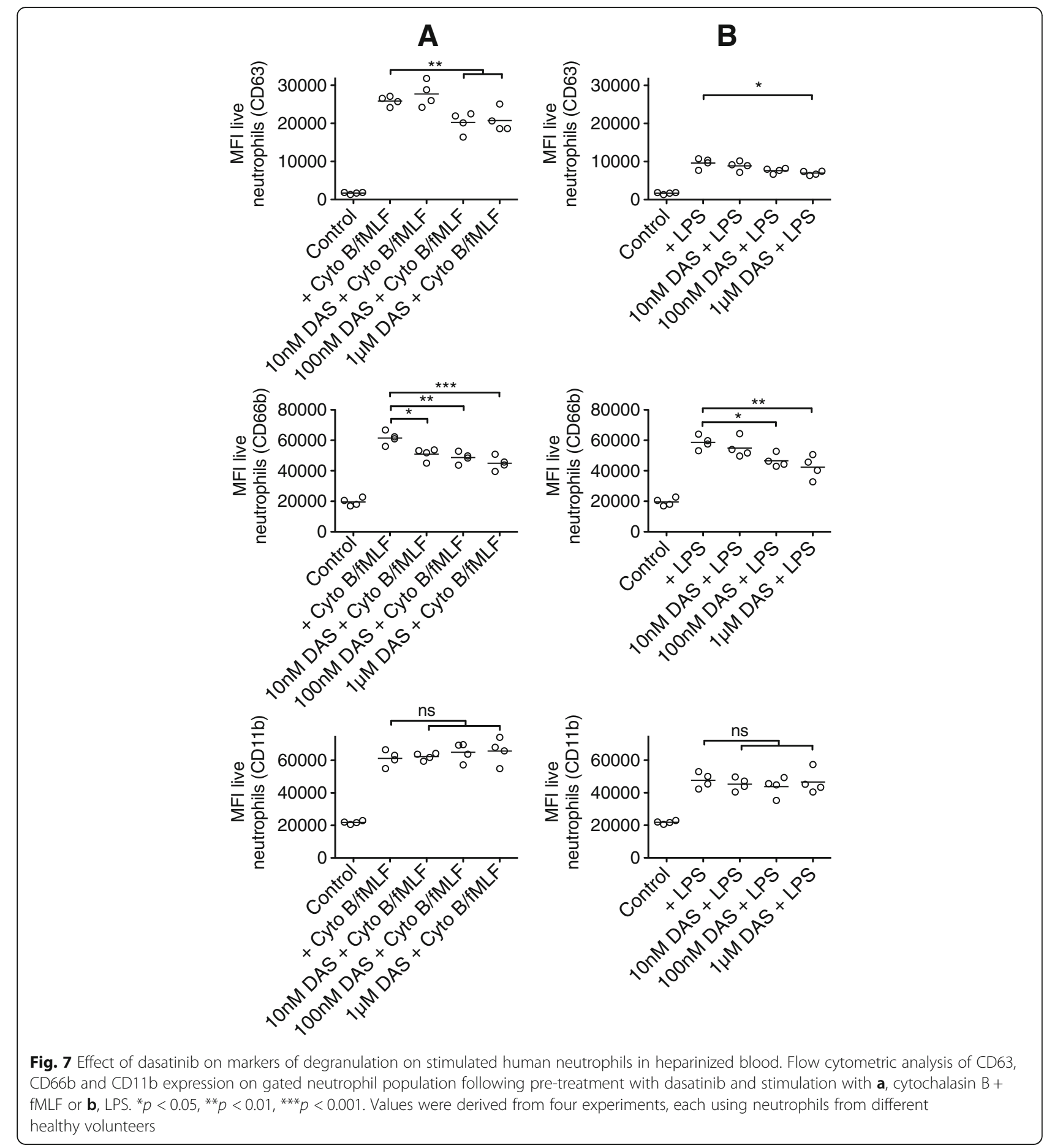

on neutrophil recruitment and migration seems unlikely to explain the observed impairment of clearance of $E$. coli. These data are in contrast to the beneficial effects of src kinase inhibition with dasatinib in the CLP sepsis model, where the lower dose of $1 \mathrm{mg} / \mathrm{kg}$ dasatinib administered $30 \mathrm{~min}$ before, $6 \mathrm{~h}$ and $24 \mathrm{~h}$ after CLP (compared with our own time course of $30 \mathrm{~min}$ before and $12 \mathrm{~h}$ after i.t. E. coli), improved survival and sepsis severity, as well as reduced intraperitoneal bacterial growth and spread [18]. Possible explanations for these differences are the timings of dasatinib administration after the acute inflammatory stimulus in the CLP model and the differing stimulus intensity between the two models. Similar to our findings, at the higher dose of $10 \mathrm{mg} / \mathrm{kg}$ dasatinib, there was a harmful effect in the CLP model. 
We believe that the in vitro inhibitory effects of dasatinib on degranulation, superoxide generation and chemotaxis are not borne out in our in vivo model of infection, where the more likely explanation for impaired bacterial clearance may relate to inhibitory effects of dasatinib on phagocytosis. Dasatinib impaired ingestion of $E$. coli, and also inhibited phagocytosis of zymosan particles in vitro. The possibility remains that phagocytosis in vivo may have been further inhibited by the impairment in neutrophil adhesion induced by dasatinib, which has also been described by others [20-22]. Phagocytosis is more efficient when neutrophils are adherent to a substratum [23] and the implication, at least in vitro, is that dasatinib impairs tethering of neutrophils to surfaces. In contrast to dasatinib's inhibition of phagocytosis, we did not observe the hypothesized marked effect on neutrophil degranulation.

In addition to suggesting a potential link to increased infection risk in patients prescribed dasatinib, our results provide an important caveat to recent studies suggesting a potential therapeutic role for src kinase inhibitors in inflammatory lung disorders. For example, src kinase inhibitors including dasatanib have been shown to significantly reduce lung injury and inflammation in animal models of acute lung injury [17, 24-26]. Similarly, beneficial anti-inflammatory effects have been attributed to dasatinib in experimental models of allergic asthma and acute silicosis $[27,28]$. The acute lung injury studies described were all notable for using chemical and/or physical stimuli (rather than infective stimuli) to produce lung damage. Our findings extend the existing literature by suggesting that src kinase inhibitors deserve further study as anti-inflammatory medications, while keeping in mind that they may exacerbate infective causes of lung inflammation, and increase the risk of secondary infection.

We believe there are several strengths to this study. These included the use of doses of dasatinib used in comparable studies in the literature, and the reproducibility of in vivo data (as reflected by similar results for our separate control groups in Figs. 1, 2, 3), which in turn increases confidence in the observed effects of dasatinib. Furthermore, we used a broad range of assays interrogating different human neutrophil functions, with a view to understanding potential relevance in the human setting.

We also recognize that there were limitations in our study. A key consideration is whether our findings are compatible with existing literature. In particular, the elegant study by Gonçalves-de-Albuquerque et al. showed that dasatinib reduced lung infection in a CLP model [18]. There were fundamental differences between the studies including strain of mouse, route of administration of dasatinib (gavage versus our i.p. route) and the model (peritoneal, polymicrobial infection with secondary lung infection versus our direct, primary lung infection). The direct model consistently yields lung parenchymal infection and milder systemic change, and we opted for i.p. administration to avoid first-pass metabolism effects. However, we acknowledge that gavage more closely mimics the oral administration in patients, and that our data cannot exclude a beneficial effect of dasatinib in lung infection secondary to severe, systemic sepsis. Our findings should therefore be considered in the specific context of acute, primary pneumonia.

Secondly, it is possible that dasatinib has subtly different effects on innate immunity in mice and in humans $[29,30]$. Our in vitro data in human neutrophils show that dasatinib inhibits degranulation from primary and secondary granules at higher concentrations, but clear evidence for a similar effect in mice was lacking. Also, pneumonia in patients is very rarely induced by a sudden bolus of infective material, as is traditionally used in mouse models of pneumonia. Furthermore, we have investigated one potential pathogen here. While noting these important limitations, we would defend the current study on the grounds that, as described above, a large prospective study of whether dasatinib is associated with pneumonia during use in patients would be extremely difficult to fund and perform at present, and no good rodent model imitating the true pathogenesis of human pneumonia is currently available to our knowledge. As such, we believed it important to perform a thorough assessment of one clearly relevant organism. We therefore conclude that our study has demonstrated proof of principle for in vivo susceptibility to pulmonary infection associated with dasatinib (and established conditions to explore this area further), but that these limitations must be kept in mind.

Third, it is extremely difficult to know whether the doses and concentrations of dasatanib used here are representative of concentrations of dasatinib in patients' lung tissue. Prospective clinical studies specifically seeking to determine whether dasatanib increases the risk of pneumonia in patients would require prohibitively large numbers of subjects, and sub-studies to determine tissue concentrations of dasatinib in the lung of infected patients would be extremely challenging. Certainly, no such studies could be funded without supportive preliminary data demonstrating proof of principle for a proinfective role of dasatanib in vivo (which we believe is provided here). Therefore, for this initial study, we selected doses used in comparable in vitro and in vivo studies.

Fourth, we acknowledge that studies of src kinase inhibitors are necessarily complicated by variations in tyrosine kinase inhibitor specificity. Dasatinib has inhibitory effects on abl kinases in addition to src kinases [31, 
32]. The spectrum of src kinase inhibition for dasatinib differs from that of other src kinase inhibitors used in cancer research, such as PP1 or PP2. More specific individual src family kinase inhibitors e.g. for Hck and Fgr are not currently available, but would theoretically represent a potential way of selectively controlling individual neutrophil granule subtype activity to maintain phagocytosis and killing functions, while attenuating harmful extracellular degranulation [33]. However we selected dasatinib precisely because of its clinical relevance. As such we consider dasatinib to be the appropriate agent for this study.

\section{Conclusions}

In summary, src kinase inhibition with dasatinib inhibits clearance of $E$. coli from the mouse lung, without influencing neutrophil recruitment. Instead, dasatinib impairs the capacity of neutrophils to adhere and to phagocytose E. coli. These data provide novel experimental evidence suggesting that src kinase inhibition is unlikely to provide an effective treatment strategy for infective acute lung injury.

\section{Methods}

\section{In vivo acute lung infection model}

C57BL/6 (female aged 8-12 weeks old; Charles River) received $1 \mathrm{mg} / \mathrm{kg}$ or $10 \mathrm{mg} / \mathrm{kg}$ dasatinib (Cambridge Bioscience, Cambridge, UK) i.p. in dimethyl sulfoxide or vehicle control. After $30 \mathrm{~min}$, animals received i.t. E. coli (serotype ATCC25922, grown to log phase) at a dose of $1 \times 10^{6} \mathrm{cfu}$ as described [34]. Dasatinib or vehicle control were re-administered i.p. at $12 \mathrm{~h}$ and samples obtained at $24 \mathrm{~h}$.

\section{Assessment of bacterial load}

At $24 \mathrm{~h}, \mathrm{BAL}$ was performed with three aliquots of $800 \mu \mathrm{l}$ sterile ice-cold saline administered i.t., and sequentially gently aspirated. $50 \mu \mathrm{l} \mathrm{BAL}$ from the first lavage was kept for flow cytometric analysis of neutrophils and bacterial determination, with the remainder centrifuged at $300 \mathrm{~g}$ for $5 \mathrm{~min}$, with cell-free supernatant from the first lavage stored at $-80^{\circ} \mathrm{C}$ for subsequent biochemical analysis. Blood samples were taken by direct cardiac puncture and added to citrate (as anticoagulant). Following lavage lungs were perfused with $20 \mathrm{ml}$ phosphate-buffered saline and removed en bloc prior to digestion using $5 \mathrm{mg} / \mathrm{ml}$ collagenase D (Roche) in HBSS with cations [34]. Blood, BAL fluid and homogenized lung, liver and spleen samples were all incubated overnight at $37^{\circ} \mathrm{C}$ on LB agar plates to determine bacterial colony counts.

\section{Quantification of neutrophils}

After collagenase digestion of the right lung, red blood cells were lysed (ACK lysis buffer, Invitrogen) and Fcmediated antibody binding reduced by incubation with Fc block (Mouse BD Fc block, BD Bioscience). Lung digest cells, BAL fluid cells and whole blood were incubated with anti-CD45 and anti-Ly6G antibodies (Biolegend, London, UK) for $30 \mathrm{~min}$ at $4{ }^{\circ} \mathrm{C}$ in the dark. After addition of FACSlyse (BD Bioscience) cells were resuspended in PBS and $50 \mu \mathrm{l}$ flow-check fluorospheres added (Beckman Coulter, Brea, CA) prior to flow cytometric analysis.

\section{Assays of neutrophil degranulation in vivo}

BAL fluid cell samples were incubated with anti-Ly6G, anti-CD63 and anti-CD11b antibodies (Biolegend, London, UK), with flow analysis of neutrophil populations. CD63 is a membrane protein expressed on azurophilic (primary) granules and CD11b/CD18 (Mac-1) is a $\beta 2$ integrin expressed across specific (secondary), gelatinase (tertiary) and secretory vesicles [35]. Both can be used as surrogate markers of degranulation when these granules fuse with the extracellular membrane [36]. BAL fluid MPO activity was quantified using the tetramethylbenzidine (TMB)/hydrogen peroxide $\left(\mathrm{H}_{2} \mathrm{O}_{2}\right)$ colorimetric assay [37], while MPO concentration was quantified using a mouse MPO enzyme-linked immunosorbent assay (ELISA) kit (R\&D Systems, Abingdon, UK).

\section{Quantification of BAL cytokines and alveolar protein concentration}

The pro- and anti-inflammatory cytokine profile of BAL fluid was quantified using ELISA kits for $\mathrm{KC}$, monocyte chemoattractant protein-1 (MCP-1), TNF and interleukin (IL)-10 (R\&D Systems, Abingdon, UK) according to the manufacturers' instructions. Total protein was measured using a Pierce bicinchoninic acid assay kit (Life Technologies, Paisley, UK) and BAL fluid IgM by ELISA (eBioscience, Hatfield, UK).

\section{Quantification of systemic toxicity}

Plasma alanine transaminase (ALT) and lactate were quantified according to manufacturers' instructions (Alpha Laboratories, Eastleigh, UK and Randox Laboratories, Crumlin, UK respectively) and adapted for use on a Cobas Fara centrifugal analyzer (Roche Diagnostics, Burgess Hill, UK).

\section{Isolation of neutrophils from human blood}

Neutrophils were isolated from the citrated whole blood of healthy donors by dextran (Pharmacosmos, Holbaek, Denmark) sedimentation and fractionation through isotonic discontinuous Percoll (GE Healthcare, Little Chalfont, UK) gradients, as described [38, 39]. Neutrophils 
were re-suspended at assay-dependent concentrations in warmed Iscove's modified Dulbecco's medium (IMDM, Life Technologies, Paisley, UK) with 1\% autologous serum. Only preparations yielding $>95 \%$ neutrophil purity with $>95 \%$ viability were used in experiments.

\section{Neutrophil phagocytosis assay}

Human neutrophils adherent to tissue culture plastic were exposed to autologous serum-opsonized $\mathrm{pH}$ sensitive pHrodo $^{\mathrm{ma}}$ green E. coli Bioparticles for $30 \mathrm{~min}$ at $37^{\circ} \mathrm{C}$ (Life Technologies, Paisley, UK), before analyzing fluorescence at $585 \mathrm{~nm}$.

In separate assays, neutrophils were exposed for 30 min at $37^{\circ} \mathrm{C}$ to zymosan particles (derived from Saccharomyces cerevesiae) that had been opsonized using autologous serum. Phagocytosis of zymosan particles was quantified as described [40].

\section{Neutrophil adhesion assay}

Neutrophil adhesion to plastic was adapted from previously described methods [41]. Briefly, fresh neutrophils in IMDM were added to a 24-well plate with $50 \mathrm{nM}$ fMLF (Sigma, Gillingham, UK) for $60 \mathrm{~min}$ to promote adhesion. Non-adherent cells were washed off before adherent cells were trypsinized, suspended with CountBright $^{\mathrm{m}}$ Absolute Counting Beads (Life Technologies, Paisley, UK) and counted by flow cytometric analysis.

\section{Neutrophil chemotaxis assay}

Two-dimensional 'under agarose' chemotaxis towards fMLF was measured as described [42].

\section{Neutrophil superoxide assay}

The extracellular release of superoxide anion by neutrophils in response to priming by platelet-activating factor (PAF, Sigma, Gillingham, UK) and activation by fMLF was quantified using the cytochrome $\mathrm{C}$ reduction assay [43].

\section{In vitro neutrophil degranulation assays}

Fresh heparinized blood samples were stimulated with $10 \mu \mathrm{M}$ cytochalasin B (Sigma, Gillingham, UK) $/ 1 \mu \mathrm{M}$ fMLF or E. coli 026:B6-derived LPS (Sigma, Gillingham, UK) to induce neutrophil degranulation before incubating with anti-CD63, anti-CD66b (localized on secondary neutrophil granules) and anti-CD11b antibodies for flow analysis, as described [36].

\section{Neutrophil toxicity assays}

Direct cytotoxicity of dasatinib on isolated neutrophils was quantified using a Roche LDH cytotoxicity kit (Roche, Sussex, UK), according to the manufacturer's instructions. Cytocentrifuge preparations of isolated neutrophils exposed to dasatinib for $24 \mathrm{~h}$ and stained with
Giemsa (Sigma, Gillingham, UK) were assessed for morphological changes consistent with apoptosis.

\section{Statistical analysis}

Flow cytometry data were analyzed using Flojo version 10.0.7 (Ashland, OR, US). Results, expressed as individual data points with mean \pm s.d. (in vivo data) or mean (in vitro data), were analyzed using GraphPad Prism (Graphpad Software, La Jolla, CA, US), by repeated measures one-way analysis of variance or a Friedman test with a post-hoc Dunn's test, or an unpaired t-test, as appropriate. Statistical significance was considered to be at the level of $p<0.05$.

\section{Supplementary information}

Supplementary information accompanies this paper at https://doi.org/10. 1186/s12950-020-00261-5.

Additional file 1 Intratracheal instillation of E. coli resulted in the anticipated time-dependent accumulation of neutrophils in the pulmonary interstitium and alveolar space, with marked inflammation present at $24 \mathrm{~h}$, and near resolution by $48 \mathrm{~h}$ (Supplementary Fig. 1). Dasatinib alone, without i.t. E. coli, caused no cellular influx into the alveolar space or alveolar protein leak (Supplementary Fig. 2). When E. coli-infected mice were treated with either dasatinib or control at 0 and $12 \mathrm{~h}$, small mean increments were seen in BAL fluid concentrations of the pro-

inflammatory cytokines KC and TNF in association with dasatinib at 10 $\mathrm{mg} / \mathrm{kg}$ (Supplementary Fig. 3), but no differences were observed in the numbers of neutrophils in the pulmonary interstitium (Supplementary Fig. 4). A small increase in blood neutrophils was observed in association with the higher dose of dasatinib studied $(10 \mathrm{mg} / \mathrm{kg}$ ) (Supplementary Fig. 4). Treatment of E. coli-infected mice resulted in a significant increase in blood levels of lactate and of the hepatic enzyme ALT (Supplementary Fig. 5). When dasatinib or control was applied directly to isolated human blood neutrophils, no difference was observed in the release of LDH or on the rate of neutrophil apoptosis (Supplementary Fig. 6). Figure S1 Kinetics of neutrophil influx into the lung interstitium and alveolar space following i.t. instillation of $E$. coli in mice. $n=2-3$ mice per time point.

Figure S2 Effect of dasatinib pre-treatment alone on cellular influx to the alveolar space and alveolar protein leak. $n=1$ mouse per condition. Figure S3 Effect of dasatinib pre-treatment on the pro/anti-inflammatory cytokine profile of the alveolar space. A, KC, B, MCP-1, C, TNF and D, IL10 concentrations in BAL fluid from mice exposed to i.t. E. coli. ${ }^{*} p<0.05$, ${ }^{* *} p<0.01,{ }^{* * *} p<0.0001$. Values were derived from two experiments (1 $\mathrm{mg} / \mathrm{kg}$ or $10 \mathrm{mg} / \mathrm{kg}$ dasatinib vs. control administered at 0 and $12 \mathrm{~h}$ ) using 8 mice per group. Figure $\mathbf{S 4}$ Effect of dasatinib pre-treatment on blood neutrophilia and neutrophil influx into lung interstitium. A, \% neutrophils of all leukocytes in blood and $\mathbf{B}$, total number of neutrophils in lung homogenates from mice exposed to i.t. E. coli. ${ }^{*} p<0.05$. Values were derived from two experiments $(1 \mathrm{mg} / \mathrm{kg}$ or $10 \mathrm{mg} / \mathrm{kg}$ dasatinib vs. control administered at 0 and 12 h) using 8 mice per group. Figure S5 Effect of dasatinib pre-treatment on markers of extrapulmonary toxicity. A, plasma lactate and $\mathbf{B}$, plasma ALT concentrations in mice exposed to i.t. E. coli. ${ }^{* *} p<0.01,{ }^{* * *} p<0.0001$. Values were derived from two experiments (1 $\mathrm{mg} / \mathrm{kg}$ or $10 \mathrm{mg} / \mathrm{kg}$ dasatinib vs. control administered at 0 and $12 \mathrm{~h}$ ) using 8 mice per group. Figure $\mathbf{S 6}$ Effect of dasatinib on the viability and apoptosis of isolated human blood neutrophils. A, cytotoxicity of dasatinib, as measured by extracellular $\mathrm{LDH}$ release, expressed as a percentage of the sample lysed using Triton. B, apoptosis rates after $24 \mathrm{~h}$ of culture with PMA (phorbol myristate acetate) or dasatinib, as measured by cell morphological characteristics under light microscopy. ${ }^{* *} p<0.01$. Values were derived from three (A) or four (B) experiments, each using neutrophils from different healthy volunteers.

\section{Acknowledgements}

Professor Simpson is a National Institute for Health Research (NIHR) Senior Investigator. The views expressed in this article are those of the authors and 
not necessarily those of the NIHR, or the Department of Health and Social Care.

\section{Authors' contributions}

$J G M, D A D, M H R S, J S, C D L, A G R$, and AJS conceived and planned the experiments. JGM performed the in vitro experiments. JGM, DAD and CDL performed the in vivo experiments. MHRS and JS contributed to sample preparation. JGM and DAD analyzed the data. JGM, DAD, MHRS, JS, CDL, AGR, and AJS all contributed to interpretation of data. JGM and AJS wrote the final manuscript with input from all authors. The author(s) read and approved the final manuscript.

\section{Funding}

This study was funded by the Wellcome Trust WT095157 (JGM), WT096497 (DAD) and WT094415 (CDL), UK Medical Research Council MR/K013386/1 (AGR).

\section{Availability of data and materials}

The datasets used and/or analysed during the current study are available from the corresponding author on reasonable request.

\section{Ethics approval and consent to participate}

All in vivo experiments were performed in accordance with UK Home Office regulations (project licence number PPL 60-4531). Approval to obtain whole blood from healthy volunteers was provided by the Newcastle and North Tyneside Research Ethics Committee (reference number 12/NE/0121).

\section{Consent for publication}

Not applicable.

\section{Competing interests}

The authors declare that they have no competing interests.

\section{Author details}

${ }^{1}$ Translational and Clinical Research Institute, Newcastle University, Newcastle upon Tyne NE2 4HH, UK. ${ }^{2}$ Centre for Inflammation Research, Queen's Medical Research Institute, University of Edinburgh, Edinburgh EH16 4TJ, UK.

Received: 11 June 2020 Accepted: 30 September 2020

\section{Published online: 30 October 2020}

\section{References}

1. Parsons PE, Fowler AA, Hyers TM, Henson PM. Chemotactic activity in bronchoalveolar lavage fluid from patients with adult respiratory distress syndrome. Am Rev Respir Dis. 1985;132(3):490-3.

2. Weiland JE, Davis WB, Holter JF, Mohammed JR, Dorinsky PM, Gadek JE. Lung neutrophils in the adult respiratory distress syndrome. Clinical and pathophysiologic significance. Am Rev Respir Dis. 1986;133(2):218-25.

3. Steinberg KP, Milberg JA, Martin TR, Maunder RJ, Cockrill BA, Hudson LD. Evolution of bronchoalveolar cell populations in the adult respiratory distress syndrome. Am J Respir Crit Care Med. 1994;150(1):113-22.

4. Kolaczkowska E, Kubes P. Neutrophil recruitment and function in health and inflammation. Nat Rev Immunol. 2013;13(3):159-75.

5. Yang F, Feng C, Zhang X, Lu J, Zhao Y. The diverse biological functions of neutrophils, beyond the defense against infections. Inflammation. 2016; 210(7):1283-99.

6. Reeves EP, Lu H, Jacobs HL, Messina CGM, Bolsover S, Gabella G, et al. Killing activity of neutrophils is mediated through activation of proteases by K+ flux. Nature. 2002:416(6878):291-7.

7. Segal AW. How neutrophils kill microbes. Annu Rev Immunol. 2005;23: 197-223.

8. Nauseef WM. How human neutrophils kill and degrade microbes: an integrated view. Immunol Rev. 2007;219:88-102.

9. Grommes J, Soehnlein O. Contribution of neutrophils to acute lung injury. Mol Med. 2011;17(3-4):293-307.

10. Hogg JC. Felix Fleischner lecture. The traffic of polymorphonuclear leukocytes through pulmonary microvessels in health and disease. AJR Am J Roentgenol. 1994;163(4):769-75.

11. Mócsai A, Ligeti E, Lowell CA, Berton G. Adhesion-dependent degranulation of neutrophils requires the Src family kinases Fgr and Hck. J Immunol. 1999; 162(2):1120-6.
12. Fumagalli L, Zhang H, Baruzzi A, Lowell CA, Berton G. The Src family kinases Hck and Fgr regulate neutrophil responses to $\mathrm{N}$-formyl-methionylleucylphenylalanine. J Immunol. 2007;178(6):3874-85.

13. Kovács $M$, Németh $T$, Jakus $Z$, Sitaru $C$, Simon E, Futosi $K$, et al. The Src family kinases Hck, Fgr, and Lyn are critical for the generation of the in vivo inflammatory environment without a direct role in leukocyte recruitment. J Exp Med. 2014;211(10):1993-2011.

14. Brattås MK, Reikvam H, Tvedt THA, Bruserud $\varnothing$. Dasatinib as an investigational drug for the treatment of Philadelphia chromosome-positive acute lymphoblastic leukemia in adults. Expert Opin Investig Drugs. 2019; 28(5):411-20

15. Fenton SE, Hutchens KA, Denning MF. Targeting Fyn in Ras-transformed cells induces F-actin to promote adherens junction-mediated cell-cell adhesion. Mol Carcinog. 2015;54(10):1181-93.

16. Futosi K, Németh T, Pick R, Vántus T, Walzog B, Mócsai A. Dasatinib inhibits proinflammatory functions of mature human neutrophils. Blood. 2012; 119(21):4981-91.

17. Oliveira GP, Silva JD, Marques PS, Goncalves-de-Albuquerque CF, Santos HL, Vascocellos AP, et al. The effects of Dasatinib in experimental acute respiratory distress syndrome depend on dose and etiology. Cell Physiol Biochem. 2015;36(4):1644-58.

18. Gonçalves-de-Albuquerque CF, Rohwedder I, Silva AR, Ferreira AS, Kurz ARM, Cougoule $\mathrm{C}$, et al. The Yin and Yang of tyrosine kinase inhibition during experimental Polymicrobial Sepsis. Front Immunol. 2018;9:901.

19. Liau DF, Yin NX, Huang J, Ryan SF. Effects of human polymorphonuclear leukocyte elastase upon surfactant proteins in vitro. Biochim Biophys Acta. 1996;1302(2):117-28.

20. Giagulli C, Ottoboni L, Caveggion E, Rossi B, Lowell C, Constantin G, et al. The Src family kinases Hck and Fgr are dispensable for inside-out, chemoattractant-induced signaling regulating beta 2 integrin affinity and valency in neutrophils, but are required for beta 2 integrin-mediated outside-in signaling involved in sustained a. J Immunol. 2006;177(1):604-11.

21. Shattil SJ, Kim C, Ginsberg MH. The final steps of integrin activation: the end game. Nat Rev Mol Cell Biol. 2010;11(4):288-300.

22. Herter J, Zarbock A. Integrin regulation during leukocyte recruitment. J Immunol. 2013;190(9):4451-7.

23. Dupuy AG, Caron E. Integrin-dependent phagocytosis - spreading from microadhesion to new concepts. J Cell Sci. 2008;121(11):1773-83.

24. Khadaroo RG, He R, Parodo J, Powers KA, Marshall JC, Kapus A, et al. The role of the Src family of tyrosine kinases after oxidant-induced lung injury in vivo. Surgery. 2004;136(2):483-8.

25. Severgnini M, Takahashi S, Tu P, Perides G, Homer RJ, Jhung JW, et al. Inhibition of the Src and Jak kinases protects against lipopolysaccharideinduced acute lung injury. Am J Respir Crit Care Med. 2005;171(8):858-67.

26. Lee HS, Moon C, Lee HW, Park E-M, Cho M-S, Kang JL. Src tyrosine kinases mediate activations of NF-kappaB and integrin signal during lipopolysaccharide-induced acute lung injury. J Immunol. 2007;179(10): 7001-11.

27. Cruz FF, Horta LFB, de Maia LA, Lopes-Pacheco M, da Silva AB, Morales MM, et al. Dasatinib reduces lung inflammation and fibrosis in acute experimental silicosis. PLoS One. 2016;11(1):e0147005.

28. da Silva AL, Magalhães RF, Branco VC, Silva JD, Cruz FF, Marques PS, et al. The tyrosine kinase inhibitor dasatinib reduces lung inflammation and remodelling in experimental allergic asthma. Br J Pharmacol. 2016;173(7):1236-47.

29. Mestas J, Hughes CCW. Of mice and not men: differences between mouse and human immunology. J Immunol. 2004;172(5):2731-8.

30. Zschaler J, Schlorke D, Arnhold J. Differences in innate immune response between man and mouse. Crit Rev Immunol. 2014;34(5):433-54.

31. Lombardo $L$, Lee FY, Chen P, Norris D, Barrish JC, Behnia K, et al. Discovery of N-(2-Chloro-6-methyl- phenyl)-2-(6-(4-(2-hydroxyethyl)-piperazin-1-yl)-2methylpyrimidin-4- ylamino)thiazole-5-carboxamide (BMS-354825), a dual $\mathrm{Src} / \mathrm{Abl}$ kinase inhibitor with potent antitumor activity in preclinical assays. J Med Chem. 2004;47(27):6658-61.

32. Olivieri A, Manzione L. Dasatinib: a new step in molecular target therapy. Ann Oncol Off J Eur Soc Med Oncol. 2007;18(Suppl 6):vi42-6.

33. Tintori C, Laurenzana I, La Rocca F, Falchi F, Carraro F, Ruiz A, et al. Identification of Hck inhibitors as hits for the development of antileukemia and anti-HIV agents. ChemMedChem. 2013;8(8):1353-60.

34. Lucas CD, Dorward DA, Tait MA, Fox S, Marwick JA, Allen KC, et al. Downregulation of $\mathrm{Mcl}-1$ has anti-inflammatory pro-resolution effects and 
enhances bacterial clearance from the lung. Mucosal Immunol. 2014;7(4): 857-68.

35. Faurschou M, Borregaard N. Neutrophil granules and secretory vesicles in inflammation. Microbes Infect. 2003;5(14):1317-27.

36. Naegelen I, Beaume N, Plançon S, Schenten V, Tschirhart EJ, Bréchard S. Regulation of neutrophil degranulation and cytokine secretion: a novel model approach based on linear fitting. J Inflamm Res. 2015;2015: 817038.

37. Palić $D$, Andreasen $C B$, Menzel BW, Roth JA. A rapid, direct assay to measure degranulation of primary granules in neutrophils from kidney of fathead minnow (Pimephales promelas Rafinesque, 1820). Fish Shellfish Immunol. 2005;19(3):217-27.

38. Haslett C, Guthrie LA, Kopaniak MM, Johnston RB, Henson PM. Modulation of multiple neutrophil functions by preparative methods or trace concentrations of bacterial lipopolysaccharide. Am J Pathol. 1985;119(1): 101-10.

39. Dorward DA, Felton JM, Robb CT, Craven T, Kipari T, Walsh TS, et al. The cyclin-dependent kinase inhibitor AT7519 accelerates neutrophil apoptosis in sepsis-related acute respiratory distress syndrome. Thorax. 2017;72(2):182-5.

40. Conway Morris A, Kefala K, Wilkinson TS, Dhaliwal K, Farrell L, Walsh T, et al. C5a mediates peripheral blood neutrophil dysfunction in critically ill patients. Am J Respir Crit Care Med. 2009;180(1):19-28.

41. Clark SC, Dark JH, Kirby JA. An assay of neutrophil adhesion to fibronectin and its attenuation by pentoxifylline and nitric oxide. Biochem Soc Trans. 1997;25(2):1995.

42. Nelson RD, Quie PG, Simmons RL. Chemotaxis under agarose: a new and simple method for measuring chemotaxis and spontaneous migration of human polymorphonuclear leukocytes and monocytes. J Immunol. 1975; 115(6):1650-6.

43. Kitchen E, Rossi AG, Condliffe AM, Haslett C, Chilvers ER. Demonstration of reversible priming of human neutrophils using platelet-activating factor. Blood. 1996:88(11):4330-7.

\section{Publisher's Note}

Springer Nature remains neutral with regard to jurisdictional claims in published maps and institutional affiliations.

Ready to submit your research? Choose BMC and benefit from:

- fast, convenient online submission

- thorough peer review by experienced researchers in your field

- rapid publication on acceptance

- support for research data, including large and complex data types

- gold Open Access which fosters wider collaboration and increased citations

- maximum visibility for your research: over $100 \mathrm{M}$ website views per year

At $\mathrm{BMC}$, research is always in progress.

Learn more biomedcentral.com/submissions 